DR ERIK SELIM LUNDKVIST (Orcid ID : 0000-0002-2869-8995)

Article type : Original Article

\title{
The temporal relations across burnout dimensions in athletes
}

Erik Lundkvist ${ }^{1,2}$, Henrik Gustafsson ${ }^{3}$, Paul Davis ${ }^{4}$, Stefan Holmström ${ }^{4}$, Nicolas Lemyre ${ }^{5}$ \& Andreas Ivarsson ${ }^{6}$

${ }^{1}$ Department of Child- and Youth Studies, Stockholm University, Sweden; ${ }^{2}$ Department of Geography and Sustainable Development, University of St Andrews; ${ }^{3}$ Department of Health and Environmental Sciences, Karlstad University, Sweden; ${ }^{4}$ Department of Psychology, Umeå University, Sweden; ${ }^{5}$ Department of Coaching and Psychology, Norwegian School of Sport Sciences; ${ }^{6}$ School of Health and Welfare, Halmstad University, Sweden

Communicating author: es13@st-andrews.ac.uk

\begin{abstract}
Burnout is a construct that has garnered considerable attention in sport psychology within recent years. Several hypothesized models regarding how the three dimensions (exhaustion, devaluation, and reduced sense of accomplishment) temporally relate to each other have been advanced. One proposal outlined by Maslach and Leiter suggests that exhaustion predicts devaluation which predicts reduced sense of accomplishment. However, there is not consensus amongst researchers as it has been argued that exhaustion predicts devaluation and reduced accomplishment separately. The aim of this paper was to test multiple alternative hypotheses regarding the relationships of the burnout dimensions in athletes. Two samples of Swedish youth elite athletes with differing time-spans between measurements were used. Specifically, one sample involved time intensive measures collected every week over an eight week period, the other sample included four measurement points across an 18 month period. Results showed that none of the previously proposed models outlining the burnout dimensions temporal relations were supported. Statistical analysis of the models including the cross lagged predictions of dimensions did not have any statistically significant impact except when exhaustion negatively predicted devaluation between time one (month zero) and time two (month six) in the 18 month sample; this relation faded in the following timepoints. Further, issues regarding the stability of devaluation and reduced sense of accomplishment emerged as their autocorrelation were very weak in the time intensive sample. These findings raise a number of points for further theoretical and practical discussions about the athlete burnout construct.
\end{abstract}

The concept of burnout has been thoroughly researched in sport psychology over the

last three decades. ${ }^{1,2}$ However, despite advances in the understanding of the antecedents and This article has been accepted for publication and undergone full peer review but has not been through the copyediting, typesetting, pagination and proofreading process, which may lead to differences between this version and the Version of Record. Please cite this article as doi: $10.1111 / \mathrm{sms} .13000$

This article is protected by copyright. All rights reserved. 
outcomes of burnout, a number of aspects related to the theoretical discussion surrounding burnout in sport have been deficient. ${ }^{3}$ One issue stemming from the theoretical underpinnings of the most widely used definition of burnout ${ }^{4}$ relates to the temporal interplay of the underlying dimensions that have been identified as being central to the process of individuals burning out. ${ }^{5,6}$ Although two recent studies conducted within youth sports contexts have studied these relations ${ }^{7,8}$ consensus has not been reached, and understanding of the how burnout dimensions in sport relate temporally remains incomplete. Therefore, this study aims to further examine the temporal relations across burnout dimensions in competitive adolescent athletes.

Burnout, both in occupational and sport research, is considered to be a stress related syndrome. ${ }^{4,5,9}$ The burnout phenomena was originally identified in health institution workers when two researchers independent of each other saw that long term stress in workers had serious mental health consequences. ${ }^{10}$ Burnout research in sport settings, as well as other domains, ${ }^{11,12}$ has been largely guided by the theoretical framework advanced by Maslach and colleagues. ${ }^{4,10}$ Specifically, emotional exhaustion is proposed to reflect the stress response aspect of burnout and is expressed by mental exhaustion; it is described as the first symptom to manifest in the burnout process. Depersonalization and cynicism are the second dimensions identified; they differ in regards to whether job roles have direct contact with recipients (e.g., nurse or classroom teacher) or relations are not central to job responsibilities (e.g., administrative office work). Depersonalization is defined as a coping strategy employed when experiencing mental exhaustion and reflects a distancing from one's recipients. ${ }^{4}$ Cynicism is also aimed at coping with mental exhaustion, however distance from duties associated with the job role are sought. ${ }^{4}$ Finally, reduced sense of accomplishment is characterized by a perception of decreasing performance on tasks at work; it is considered the third symptom in the burnout syndrome. ${ }^{13}$ 
In consideration of Maslach's definition of burnout and the Maslach Burnout Inventory (MBI), ${ }^{13}$ a sport specific self-report measure has been developed (i.e., Athlete Burnout Questionnaire). ${ }^{14,15}$ The sport specific definition of exhaustion reflects both mental and physical exhaustion and is referred to as emotional/physical exhaustion. Sport devaluation corresponds to depersonalization/cynicism and outlines that the sport previously of central importance in the person's life is now less important than formerly reported. Reduced sense of sport accomplishment corresponds with reduced accomplishment and reflects an athlete's feeling that he/she is no longer performing to a level that correspond with his/her own expectations. ${ }^{14}$ The ABQ is by far the most widely used measure of athlete burnout. ${ }^{2,16}$

Several differing hypotheses about how the three burnout dimensions relate to each other temporally have been discussed. ${ }^{5,6,17}$ One proposal advanced by Leiter and Maslach (1988) describes a temporal process where the dimensions occur in response to each other in a set order; specifically, emotional exhaustion leads to depersonalization/cynicism, which in turn leads to a reduced sense of accomplishment. ${ }^{5,9}$ Further, Leiter and Maslach suggest exhaustion is a response to occupational stress and is the first symptom to manifest.

Thereafter, the individual attempts to cope with her/his exhaustion by detaching from work related duties and/or stressors (e.g., patients, students) depending on the occupational setting in which they are employed; consequently, exhaustion is proposed to predict depersonalization/cynicism. When a person attempts to cope with his or her exhaustion, with depersonalization/cynicism, it is anticipated that they begin to appraise accomplishments at work less positively. ${ }^{9}$ Golembiewski and colleagues ${ }^{17}$ contend that depersonalization would be the first link in a temporal chain associated with coping strategies observed in work settings. This proposal suggests that detaching from work leads to diminished performance, which can subsequently predict feelings of exhaustion. ${ }^{17}$ An alternate model of the progression of burnout posits emotional exhaustion predicts depersonalization and personal 
accomplishment separately. ${ }^{5}$ In testing three of the proposed models and one merged cross lagged model, Taris, Le Blanc, Schaufeli, and Schreurs ${ }^{6}$ observed temporal relations between burnout subscales were statistical significance (however of little practical importance) was reached for the relations between exhaustion and depersonalization, depersonalization and emotional exhaustion and, depersonalization and personal accomplishment. To sum up, these findings suggest that several models from previous studies seem to work and that one sole model of temporal relations between burnout dimensions could not be found.

Two recent studies conducted in sport settings examined the progression of burnout dimensions in relation to time. ${ }^{7,8}$ One study by Martinent, Louvet and Decret $^{8}$ used a latent class growth analysis with three data points, collections over two demanding months of sport activity, three distinct classes of trajectories were obtained for each burnout dimension. In comparing the classes, obtained from each of the three dimensions, it did not appear that the dimensions of burnout developed in tandem. However upon closer scrutiny, high levels of reduced sense of accomplishment measured at time one appeared to increase the likelihood of belonging to classes with downstream changes in the two other dimensions. ${ }^{8}$ Another recent study by Isoard-Gautheur, Guillet-Descas, Gaudreau, and Chanal compared the progression of the dimensions in adolescent athletes over a three-year period and reported a different pattern. ${ }^{7}$ Specifically, during times when emotional exhaustion was increasing sport devaluation concurrently increased. Further, at time points when sport devaluation increased, emotional exhaustion appeared to decrease. ${ }^{7}$ One possible explanation for the differing results observed between the studies relates to research design; specifically, the timing between data collection may have influenced findings. In Martinent and colleagues' study the time span between the first and last measurement was two months and in Isoard-Gautheur et al.'s study the measurement time points spanned a period up to two years. These timing differences could, at least, theoretically tap different types of processes. Additionally, neither of the 
studies undertook statistical analyses using exhaustion to predict devaluation, nor did they use devaluation to predict reduced sense of sport accomplishment; adopting these approaches would have been in line with the different hypotheses formulated by Leiter and Maslach, Golembiewsky or Taris, Le Blanc, Schaufeli and Schreurs.

The process underlying the development of burnout is of particular interest in burnout research. ${ }^{18}$ Knowledge about the progression of burnout is important as it can inform the construction and use of preventative interventions. ${ }^{19}$ Although Maslach and colleagues (1988) are specific in proposing the processes that eventually lead to burnout, details regarding how and when this processes manifests within a timeframe are unclear. ${ }^{20}$ It is widely understood that burnout develops from long-lasting work related stress; however, the term "long-lasting" is not well defined. Although the duration of time between measurement points has varied within burnout research, there is limited discussion of how the influence of time is accounted for when testing the temporal relationships across burnout dimensions. Therefore, there is a need to assess the role of time in the development of burnout and establish a clear conceptual foundation to promote understanding of the interrelationship among the three dimensions of burnout over time.

The lack of consensus and consistent research findings detailing the temporal relations of burnout dimensions in sport and other domains suggests that further study is warranted. Moreover, consideration of the temporal relations in predicting subsequent burnout dimensions has not fully guided studies conducted in the sport environment. Specifically, investigating highly competitive youth athletes is of particular interest as they experience intense physical demands and psychological pressure in attempting to realize their goal of becoming an elite athlete. ${ }^{19,21}$ The range of psychophysiological stressors inherent to competitive youth sport exposes athletes to the potential risk of experiencing burnout. Therefore, the aim of the present study is to replicate Taris et $\mathrm{al}^{6}$ within the domain of sport 
with competitive adolescent athletes, and test the temporal relations of the dimensions underlying the $\mathrm{ABQ}$ using additional measurement points, different time frames, and within person analysis. In order to accomplish this aim, two different samples have been used; the first is comprised of weekly measures taken over eight consecutive weeks, the second contains data collected at four measurement points six months apart over a span of one and a half years (i.e., 18 months).

\section{Method}

The data comprising the present study are derived from two samples with different intervals regarding the time between measurement points. The first sample encompasses an eight week timeframe with weekly measures, and the second sample incorporates an 18 month timeframe with data collection every six months. Both timeframes were chosen based on convenience and are parts of larger studies where additional data have been collected to answer alternative research questions. The specific burnout data analyzed in the present study have not previously reported.

\section{Participants}

Sample One. Initially 179 participants were recruited to the study and provided data at the first data collection time point. As a result of attrition and missed data collections 168 athletes were included in the analyses. The athletes comprising the sample included cross country skiers and ski-orienteers from the Swedish national youth elite program, as well as Swedish football players competing at the youth elite level. The athletes used in the subsequent data analyses were 16 to 19 years of age $(M=17.03 ; S D=.99) ; 70$ were girls and 98 were boys. Informed written consent was provided by all athletes participating in the study.

Sample 2. Initially 97 participants were recruited and completed the questionnaire at the first data collection time point; however, 17 were excluded from analyses due to missing data in 
which they failed to complete questionnaires at two time points across the data collection period. Participants were athletes from the Swedish national youth elite program competing within individual $(\mathrm{N}=63)$ and team $(\mathrm{N}=12)$ sports, 22 persons did not specify their sport. At the time of the first measurement all athletes were 16 to 18 years old, 33 were girls and 40 boys and 24 did not specify their gender. The sample ranged from regional level to international youth elite level. All participants provided informed written consent prior to participating.

\section{Instrument}

Athlete Burnout Questionnaire. ${ }^{16}$ The Swedish translation of ABQ was used to measure athlete burnout. Although contextually adapted and validated in sport contexts, ${ }^{14}$ the ABQ stems from the same definition of burnout as the Maslach Burnout Inventory (MBI) ${ }^{22}$ and items are framed for use in sport. The ABQ has three subscales: emotional/physical exhaustion, sport devaluation, and reduced sport accomplishment; each measured with five items. Specifically, emotional/physical exhaustion reflects both the mental and the physical aspects of the burnout construct (e.g., "I feel so tired from my training that I have trouble finding energy to do other things" \& "Ifeel 'wiped out' from sport". ${ }^{14}$ Sport devaluation reflects feelings associated with sport participation and one's own performance being of less importance than previously (e.g., "The effort I spend in sport would be better spent doing other things" \& “I don't care as much about my sport performance as I used to". ${ }^{14}$ Reduced sport accomplishment corresponds with the reduced sense of accomplishment dimension of the $\mathrm{MBI}^{14,23}$ and reflects feelings of when a person no longer perform to a level that corresponds with his/her own expectations (e.g., "It seems that no matter what I do, I don't perform as well as I should"). ${ }^{14}$ Respondents score each item on a five point scale $(1=$ almost never, $2=$ rarely, $3=$ sometimes, $4=$ frequently, and $5=$ almost always). Across the questionnaire, 13 items are negatively worded and two are worded positively; the two 
positively worded items are reversed in subsequent analyses to determine a score where a greater frequency indicates higher levels of athlete burnout across the three dimensions.

\section{Procedure}

In sample one the questionnaires were distributed every week for eight consecutive weeks. The participants completed the questionnaire in a classroom setting or at a training session under the supervision of a teacher or a coach. In order to ensure confidentiality, upon completing the measures athletes placed the questionnaires in a sealed envelope and the teacher/coach had no access to the athletes' data. In sample two the questionnaires were distributed as websurveys from teachers that were responsible for the respective sport at the school. The teachers had no access to the athletes' data. Participation was optional for all participants and they were informed that they could skip questions they did not want to answer or discontinue their participation at any time.

\section{Statistical analysis}

Data were analyzed using Mplus (version 7.4). Multivariate Latent Curve Model with Structured Residuals (LCM-SR) was used to analyze data with maximum likelihood robust (MLR) estimation; this is a structural equation model (SEM) that takes into account both between person and within person relations between variables over time. ${ }^{24}$ To handle missing data full information maximum likelihood (FIML) was used. FIML is an method that uses an individually based likelihood function based on the variables that are available in the dataset. $^{25}$ To handle if missing was at random paired sample T-tests were conducted at time point one where the missing group was defined as $50 \%$ missed data points in sample one and having any missing values in sample two. No statistically significant differences between groups were found.

Because of small sample sizes three models for each sample was tested (see example of the theoretical model in Figure 1) separately. In the tested model we estimated a full 
bidirectional model with cross-lagged paths in both directions; this approach reflects the consideration that the relations of all three dimensions in the burnout construct may plausibly occur during shorter periods of time without being directly attributed to long term stress (as outlined in models describing the burnout syndrome). ${ }^{4}$ For example, intensive periods of training or matches could possibly induce feelings of exhaustion; however, these acute feelings may dissipate if coaches and athletes manage them effectively. This process of training and recovery is inherent to competitive sport where training load and challenging competitions are central features to athlete development. This logic can also be applied in the consideration of devaluation and reduced sport accomplishment; specifically, performances evaluated to be unsuccessful may lead to the perception of reduced sport accomplishment. However, athletes' perceptions may change if the outcome of the next competition is deemed more successful. Therefore within the accumulated scores, where exhaustion is constantly experienced over several time points (i.e., not improving or fluctuating), it is expected that the magnitude of the relation between exhaustion and devaluation should become stronger over time. As such, the scores from measurements taken at previous time points were systematically accounted for when within person relations between the constructs were analyzed. Using this approach results in an accumulated score being weighted into the computation of the temporal relations over time; for example, a relation between exhaustion and devaluation at time point seven is viewed as being stronger if previous scores were high or increasing during the preceding time points. ${ }^{24}$

To evaluate model fit, we used Chi-square $\left(\chi^{2}\right)$, comparative fit index (CFI), root mean square error of approximation (RMSEA), RMSEA with a 90\% confidence interval (RMSEACI), and Tucker-Lewis Index (TLI).Values between .90 to .95 indicate acceptable fit for CFI and TLI and greater than .95 indicate an excellent fit . For RMSEA values less than .05 
indicate good fit, values between .05 to .08 indicate acceptable fit, values between .08 to .10 indicate mediocre fit and values greater than .10 indicate unacceptable fit. ${ }^{26}$

\section{Results}

\section{Sample 1}

The results from the analyses relating to Sample 1 (i.e., the 8-week sample) are summarized in table 1 . The cross lagged model for the relation between exhaustion and devaluation showed a statistically significant $\operatorname{chi}^{2}$ value $\left(\chi^{2}=212.79, d f=118 p<.001\right)$ but acceptable fit for the other indices (RMSEA(90\% C.I.) $=.069(.05, .08)$, TLI $=0.936$ and $\mathrm{CFI}=0.937)$. The between person slope for exhaustion was zero $(\beta=0.00, p=0.956)$ and the slope for devaluation was zero $(\beta=-0.01, p=0.921)$, showing that exhaustion and devaluation, on average, did not change during this period of time (i.e., 8 weeks). Focusing on within person relations the magnitude of the cross-lagged effects varied. Exhaustion did not predict devaluation (weakest $\beta=0.04, p=0.710$; strongest $\beta=0.10, p=0.278$ ) and devaluation did not predict exhaustion (weakest $\beta=0.03, p=0.493$; strongest $\beta=0.05, p=$ 0.469). There were statistically significant autoregressive effects for exhaustion (weakest $\beta=$ $0.14, p=0.010$; strongest $\beta=0.27, p=0.004$ ) whereas devaluation was not a statistically significant predictor of devaluation (weakest $\beta=0.09, p=0.209$; strongest $\beta=0.19, p=$ 0.239). The cross-sectional relationship between the two constructs were statistically significant and varied in magnitude between the different measurement occasions (weakest $r$ $=0.25, p=0.003 ;$ strongest $r=0.50, p<0.001)$.

In the cross lagged model for where we specified the relations between devaluation and reduced sense of accomplishment the model fit indices indicating a statistically significant chi $^{2}$ value $\left(\chi^{2}=200.09, d f=120, p=<0.001\right)$ but acceptable fit for the other indices $($ RMSEA $(90 \%$ C.I. $)=0.07(0.05,0.08)$, TLI $=0.948$ and CFI $=0.949)$. The between person slope for devaluation were near zero $(\beta=0.02, p=0.476)$ as well as the slope for reduced 
sport accomplishment $(\beta=0.02, p=0.073)$. Within person relations showed that devaluation was not a statistically significant predictor of reduced sense of accomplishment (weakest $\beta=$ $-0.02, p=0.986$; strongest $\beta=0.09, p=0.404)$ and reduced sense of accomplishment was not a statistically significant predictor of devaluation (weakest $\beta=0.05, p=0.282$; strongest $\beta=0.09, p=0.295)$. There were no statistically significant autoregressive effects for devaluation (weakest $\beta=0.12, p=0.202$; strongest $\beta=0.20, p=0.248$ ) or reduced sense of sport accomplishment (weakest $\beta=0.11, p=0.133$; strongest $\beta=0.15, p=0.094$ ). Crosssectional effects for the relationship between the two constructs were statistically significant and varied between the different measurement occasions (weakest $r=0.26, p<0.001$; strongest $r=0.45, p<0.001)$.

The cross lagged model for relations between exhaustion and reduced sense of accomplishment the model fit indices for the model showed a statistically significant chi $^{2}$ value $\left(\chi^{2}=193.98, d f=117, p=<0.001\right)$ but acceptable fit for $\operatorname{RMSEA}((90 \%$ C.I $)=0.06$ $(.05, .08))$ and excellent fit for both TLI and CFI $($ TLI $=0.953$ and CFI $=0.954)$. The slope for exhaustion was close to zero $(\beta=0.00, p=0.96)$ as well as the slope for reduced sport accomplishment $(\beta=0.02, p=0.07)$. Within person relations showed that exhaustion was not a statistically significant predictor of reduced sense of accomplishment (weakest $\beta=0.07, p=$ 0.222 ; strongest $\beta=0.16, p=0.169)$ and reduced sense of accomplishment was not a statistically significant predictor of exhaustion (weakest $\beta=0.01, p=0.897$; strongest $\beta=$ $0.01, p=0.897)$. There were statistically significant autoregressive effects for exhaustion (weakest $\beta=0.14, p=0.010$; strongest $\beta=0.29, p=0.002$ ) but not for reduced sense of sport accomplishment (weakest $\beta=0.08, p=0.192$; strongest $\beta=0.13, p=0.146$ ). Crosssectional effects for the relationship between the two constructs were statistically significant and varied between the different measurement occasions (weakest $r=0.26, p<0.001$; strongest $r=0.45, p<0.001)$. 


\section{Sample 2}

The results examining sample 2, where there were six months between each of the different measurement occasions, are summarized in table 2. The model including relations between exhaustion and devaluation had problems regarding the variances for between person slopes for both exhaustion and devaluation which forced us to estimate the variance of the slope to zero for both dimensions. The model showed $\mathrm{chi}^{2}$ value that was not statistically significant $\left(\chi^{2}=29.40, d f=22, p=0.13\right)$ and acceptable fit for RMSEA (RMSEA(90 C.I.) $=$ $0.06(0.00,0.12))$ and excellent fit for TLI and CFI $($ TLI $=0.959$ and CFI $=0.968)$. The slope for exhaustion in the autoregressive model was positive but close to zero $(\beta=0.08, p=$ $0.009)$, as well as the slope for devaluation $(\beta=0.08, p=0.007)$. Within person relations showed that for exhaustion was a negative statistically significant predictor of devaluation between time one and time two but the relation was not found for the other two tested intervals $\left(\beta_{\mathrm{t} 1-2}=-0.30, p=0.033 ; \beta_{\mathrm{t} 2-3}=-0.04, p=0.717 ; \beta_{\mathrm{t} 3-4}=0.17, p=0.426\right)$ and devaluation did not predict exhaustion $\left(\beta_{\mathrm{t} 1-2}=0.03, p=0.744 ; \beta_{\mathrm{t} 2-3}=0.04, p=0.748 ; \beta_{\mathrm{t} 3-4}=\right.$ $0.05, p=0.742)$. There were no statistically significant auto regressions for exhaustion $\left(\beta_{\mathrm{t} 1-2}\right.$ $\left.=-0.07, p=0.605 ; \beta_{\mathrm{t} 2-3}=-0.05, p=0.622 ; \beta_{\mathrm{t} 3-4}=0.06, p=0.602\right)$ or devaluation $\left(\beta_{\mathrm{t} 1-2}=\right.$ $\left.0.06, p=0.659 ; \beta_{\mathrm{t} 2-3}=0.08, p=0.658 ; \beta_{\mathrm{t} 3-4}=0.09, p=0.661\right)$. Cross-sectional effects for the relationship between the two constructs varied between the different measurement occasions (weakest $r=-0.03, p=0.902$ to $r=0.68, p<0.001$ ).

The model in which devaluation and reduced sense of accomplishment were included had problems regarding the variances for between person slopes for reduced sense of accomplishment. We, therefore, fixed the variance of the slope to zero. The model fit in the redefined model was had an insignificant $\operatorname{chi}^{2}$ value $\left(\chi^{2}=27.80, d f=19, p=0.08\right)$, RMSEA showed acceptable fit $($ RMSEA $(90 \%$ C.I. $)=0.08(0.00,0.13))$ and TLI/CFI values that showed excellent fit $(\mathrm{TLI}=.960$ and CFI $=.973)$. The between person slope for devaluation 
was positive but close to zero $(\beta=0.08, p=.004)$ and the slope of reduced sense of sport accomplishment was zero $(\beta=0.00, p=.975)$. Within person relations showed that devaluation was not a statistically significant predictor of reduced sense of accomplishment $\left(\beta_{\mathrm{t} 1-2}=0.04, p=0.783 ; \beta_{\mathrm{t} 2-3}=0.06, p=0.688 ; \beta_{\mathrm{t} 3-4}=0.05, p=0.783\right)$ and reduced sense of accomplishment did not predict devaluation $\left(\beta_{\mathrm{t} 1-2}=0.20, p=0.114 ; \beta_{\mathrm{t} 2-3}=0.20, p=0.060\right.$; $\left.\beta_{\mathrm{t} 3-4}=0.22, p=0.086\right)$. There were no statistically significant auto regressive effect for devaluation $\left(\beta_{\mathrm{t} 1-2}=-0.01, p=0.956 ; \beta_{\mathrm{t} 2-3}=0.01, p=0.956 ; \beta_{\mathrm{t} 3-4}=0.02, p=0.956\right)$ or reduced sense of accomplishment $\left(\beta_{\mathrm{t} 1-2}=-0.02, p=0.870 ; \beta_{\mathrm{t} 2-3}=-0.02, p=0.873, \beta_{\mathrm{t} 3-4}=-\right.$ 0.02, $p=0.871)$. Cross-sectional effects for the relationship between the two constructs were statistically significant and varied between the different measurement occasions (weakest $r=$ $0.42, p=0.041$, strongest $r=0.63, p<0.001)$.

The model where exhaustion and reduced sense of accomplishment were included had problems because the variance for the between person slopes for exhaustion were negative. Therefore we specified the slope variance to zero for exhaustion. The redefined model showed a chi ${ }^{2}$ value that was not statistically significant $\left(\chi^{2}=21.13, d f=21, p=0.45\right)$, RMSEA indicated a good fit $(90 \%$ C.I. $)=0.01(0.00,0.09))$ and TLI/CFI showed excellent fit $(\mathrm{TLI}=0.999$ and CFI $=0.999)$. The slope for exhaustion in the autoregressive model was close to zero $(\beta=0.07$, and $p=0.02)$ and the slope for reduced sense of sport accomplishment were also close to zero $(\beta=-0.01, p=0.76)$. Within person relations show that exhaustion was not a statistically significant predictor of reduced sense of accomplishment $\left(\beta_{\mathrm{t} 1-2}=-0.11, p=0.425 ; \beta_{\mathrm{t} 2-3}=-0.03, p=0.778 ; \beta_{\mathrm{t} 3-4}=0.07, p=0.691\right)$ and reduced sense of accomplishment did not predict exhaustion $\left(\beta_{\mathrm{t} 1-2}=0.05, p=0.458 ; \beta_{\mathrm{t} 2-3}=\right.$ $\left.0.06, p=0.452 ; \beta_{\mathrm{t} 3-4}=0.06, p=0.460\right)$. There were no statistically significant within person auto regressions for exhaustion $\left(\beta_{\mathrm{t} 1-2}=-0.06, p=0.583 ; \beta_{\mathrm{t} 2-3}=-0.05, p=0.587 ; \beta_{\mathrm{t} 3-4}=-0.06\right.$, $p=0.581)$ or reduced sense of accomplishment $\left(\beta_{\mathrm{t} 1-2}=-0.10, p=0.337 ; \beta_{\mathrm{t} 2-3}=-0.14, p=\right.$ 
$\left.0.401 ; \beta_{\mathrm{t} 3-4}=-0.13, p=0.374\right)$. Cross-sectional effects for the relationship between the two constructs varied between the different measurement occasions (weakest $r=0.03, p=0.864$, strongest $r=0.34, p=0.015)$.

\section{Discussion}

The aim of the current study was to examine possible temporal relations of the dimensions underlying the $\mathrm{ABQ}$ across varying time frames and within individuals. Guided by Taris et al's. ${ }^{6}$ investigation of potential temporal relationships between the dimensions of the MBI, the present study included additional measurement points, different time frames, and within person analysis. The results emerging from the analysis of the two samples in the present study suggest that most proposed models ${ }^{4,6,9,17}$ outlining the development of burnout appears to be problematic in a sport context. In particular, the cross lagged paths for within person prediction of the subscales of the ABQ were unrelated or very weak for both samples with one exception, the negative relation between exhaustion and devaluation between time one and time two in the sample with long time frames.

In consideration of the results with a focus on hypothesis testing, the findings across the two samples did not show between person trajectories increasing over time for any of the burnout dimensions. Further, the within person analysis for both samples did not provide support for either of the tested hypotheses. In the sample with measurements taken every six months, one relation was observed where exhaustion at time one predicted decreased devaluation at time two, to then being slightly positive between time three and four. In relation to other previously tested models of burnout, the effect between time one and time two is difficult to explain and may potentially be attributed to the occurrence of a type 1 error. However, the number of missing values could also influence the results. Also, the fact that the times when data was collected could coincide with some school or sport related 
issues, especially given the instability shown in the devaluation subscale in the sample with short time between measures those explanations are plausible.

In comparing the findings of the present study with the two other studies in a sport context guided by similar research questions ${ }^{7,8}$ a consensus fails to be gained. That said, a principal clarification must be noted as neither the statistical analyses, nor the research questions are identical across the three studies. The present study is the sole examination of the temporal relations of the dimensions rather than their development alongside of each other. However, Martinent and colleagues' study did not find any relationships between the trajectories for exhaustion, devaluation, and reduced accomplishment in support for a tandem development but saw that high values of reduced sense of accomplishment was related to classes that increased over time in exhaustion and devaluation. The findings of the present study, as well as Martinet et al.'s, may support the proposal that temporal relations for burnout dimensions in sport settings are individual and diverse. ${ }^{8}$ Considering these findings in combination with the results of Isoard-Gautheur and colleagues' study, suggests that research in the context of sport does not or only partly support the model of the development of burnout dimensions forwarded by Maslach and colleagues. ${ }^{13}$ The somewhat contradictory findings in the three studies do give a base of understanding about the complexities regarding the temporality of burnout dimensions. This reinforce the suggestion that greater research examining the temporality of the burnout construct (and its dimensions) is warranted.

Interestingly, the cross lagged relations observed in the sample comprised of weekly measures over two months and the sample consisting of four measures taken over 1.5 years, do not differ (setting aside the negative relation between exhaustion and devaluation in the longer timeframe) when compared. However, the weekly autocorrelations for exhaustion seem to have an impact in the shorter timeframe, but are very weak in the sample with greater time between measure points. This could indicate that burnout dimensions do not appear 
stable over longer periods within persons, and that devaluation and reduced sense of accomplishment are observed to be unstable within measures taken weekly. Although burnout is viewed as being a stable outcome over time, this indicates that critical analysis is warranted when interpreting both longitudinal and cross-sectional studies. For example, the majority of burnout research in sport is undertaken using cross-sectional research designs, ${ }^{1,27}$ however the findings reported in these studies may not reflect the actual relations between constructs as scores of devaluation and reduced sense of accomplishment seems to fluctuate frequently and persistently.

The common practice of adapting models developed in one context (e.g., health care) and applying them to the study phenomena observed in alternative contexts can lead to contradictory findings that do not reflect the idiosyncrasies that comprise the unique context of competitive sport. The contextual differences underlying performance in youth sport and organizational settings may provide one explanation to the results of the present study. In particular, the burnout dimensions did not appear to be related over time and reduced sense of accomplishment and devaluation appeared unstable over both short and long periods of time; this observation may be related to a measurement issue as the ABQ might not provide the stability needed to reliably measures these dimensions. However, the problem can also be theoretical. Maslach's model of burnout originated from observations conducted in health care settings; although a sport specific measure (i.e., ABQ) has been used extensively in sport psychology research there are a number of shortcomings when attempting to interpret findings. ${ }^{28}$ Specifically, the ABQ is comprised of items that are framed for use in sport; however, the items from which they were derived in the MBI are applicable to work related tasks and relations. Further, the concept of exhaustion is measured with items that are framed towards feelings of tiredness caused in work settings. ${ }^{13}$ The contextual difference between work and sport are fundamental when considering that the large majority of studies in sport 
are comprised of participants that are not professionals and are not financially dependent on sport. ${ }^{28}$ Research with professional athletes may provide a sample and work place setting that is more analogous to that in which models of burnout were originally developed. Another key difference between work and sport relates to the physical demands of training and deliberate practice that may induce both mental and physical exhaustion; ${ }^{27}$ feelings of exhaustion in sport are not usually interpreted in the same manner as exhaustion associated with longstanding stress at work. Furthermore, sport devaluation and reduced accomplishment as measured by the $\mathrm{ABQ}$, address sport related performance and as such may fluctuate dramatically. That is, evaluations of performance in relation to expectations may be disproportionately influenced by recent events (i.e., the last competition or training session). Responses to items on the ABQ may reflect a different process of evaluation than the long term depersonalization/cynicism and sense of accomplishment that are framed in the MBI; therefore, the ABQ may not address burnout in the manner advanced in the original theoretical model.

Upon review, it is not necessarily implied that the ABQ is a non-valid instrument, several studies indicate that it has sound psychometric values ${ }^{16}$ and qualitative research has described how the dimensions are articulated and interpreted by athletes. ${ }^{1,19}$ However, if the results in the present study are replicated, discussion of the theoretical and practical implications of the most widely adopted definition of burnout in sports is needed. In particular, if devaluation and reduced sense of accomplishment fluctuate to the extent observed in this study it appears that the ABQ does not offer the stability required from a burnout measure from a theoretical standpoint. However in terms of being a practical tool, the ABQ's ability to monitor exhaustion may offer coaches insight into athletes' perceptions and assist in the devising training programs (e.g., periodization) and/or evaluating training load effects in athletes (e.g., tapering). Further, exhaustion related to sport specific issues remains 
an important issue in research; however, indices of exhaustion may not imply the psychological ill health associated with the burnout construct in other contexts. ${ }^{29}$ Thus further investigation of aspects (e.g., antecedents) associated with exhaustion and its implications for athletes are required.

In terms of research investigating clinical syndromes, a critical shortcoming of both the MBI and ABQ relates to their lack of clinical cut-off values; therefore, it is difficult to interpret when an individual is at risk of serious health problems. ${ }^{28,29}$ Thus, future research defining what constitutes high levels of burnout is warranted. ${ }^{16}$ In many of the burnout measures, high burnout scores might not be a threat to health to the extent that an individual will manifest fully developed burnout. ${ }^{18}$ In an attempt to avoid overstating the conclusions that can be formed from the samples comprising the present study, the individuals initially reporting higher levels of burnout subsequently report lower levels; one possible explanation of this pattern could propose that they understood their condition and took action to facilitate recovery. However, other explanations are also plausible, one could be that ABQ might have provided an inaccurate indication suggesting that the higher reported levels were problematic for the individual or that attempts to identify a sole general pattern of development in such a complex construct, where symptoms and antecedents are seen as related, might be ill advised.

In consideration of the findings of the present study, in combination with the two other related studies in sport, ${ }^{7,8}$ and studies done in occupational contexts, ${ }^{5,6,17}$ it is quite possible that Maslach and colleagues' proposed theoretical model of burnout does not offer "one size fit for all". However, this is not suggesting that burnout as a construct does not exist; rather that it is a more complex and dynamic process across individuals, domains, and time. ${ }^{30,31}$ Consequently, this deduction has implications for the design of studies examining burnout in sport; specifically, how it is defined as well as how and when it is assessed. Although the definition of burnout has been routine-like in sport psychology, clinical research has 
criticized this definition for being too narrow to be clinically relevant. ${ }^{18}$ Research investigating burnout in the sport environment may be better served by considering the use of alternative definitions of burnout that are broader and reflect greater individual variability across trajectories to stress related exhaustion issues. ${ }^{28}$ Initially, this may create some conceptual confusion; however if the concept is currently being shown to be problematic when examined from numerous approaches, in the future more thorough discussions around the definition of burnout will serve to advance burnout research in sport psychology.

\section{Limitations}

Although the present study offers potentially interesting evidence and ideas surrounding the temporal relations between burnout dimensions, several limitations must be acknowledged for cautious interpretations to be proposed. The sample size was somewhat small in both samples and warrant caution when interpreting the results. With small sample sizes missing data can have a large impact on the growth curves between persons and the within person relations of burnout relations over time. Further, running separate statistical models for each cross lagged path creates a risk for type I errors. The possibility of a gender confound also needs to be considered when interpreting our results. ${ }^{8}$

Although the temporal relations were tested with alternative statistical analyses undertaken with data collected from a greater number of time points (of different temporal intervals), it merits acknowledging that the time periods of eight weeks may be too short and/or six months too long for the optimal study of the temporal relations of the subscales of burnout. A sample comprised of monthly measures during a period of 12 months may offer an ideal level of frequency for observing these relations; however, the burnout literature does not offer concrete guidance on this proposed research design. The potential issues associated with the frequency and intensity of data collection measuring burnout requires greater study to determine the patterns underlying relations between the subscales.

This article is protected by copyright. All rights reserved. 


\section{Perspectives}

Despite the acknowledged limitations, the present study highlights that aspects of the theoretical underpinnings of the burnout construct in the sport context are problematic; guidance for further research and discussion of the implications of the theory in practice are offered. In review of the present study in combination with previous research, ${ }^{7,8}$ we suggest the conceptualization of burnout comprised of the three dimensions of exhaustion, devaluation, and reduced sense of accomplishment appears misguided because of two reasons, 1) evidence do not seem to support that the dimensions are related to the extent they should when calling a construct a syndrome and 2) devaluation and reduced sense of accomplishment seem unstable which goes against the idea of burnout being a stable construct. The routine-like use of burnout as an indicator of ill psychological health therefore requires further consideration in the design of future research studies.

\section{References}

1. Goodger K, Gorely T, Lavallee D, Harwood C. Burnout in Sport : A Systematic Review Background to Theoretical Conceptualizations of Burnout in Sport. Sport Psychol. 2007;21(2):127-151.

2. Gustafsson H, Hancock DJ, Côté J. Describing citation structures in sport burnout literature: A citation network analysis. Psychol Sport Exerc. 2014;15(6):620-626. doi:10.1016/j.psychsport.2014.07.001.

3. Lundkvist E, Gustafsson H, Davis PA. What is missing and why it is missing from coach burnout research. In: Davis PA, ed. The Psychology of Effective Coaching and Management. 1st ed. New York, NY: Nova Science Publishers; 2015:407-427. https://mail.google.com/mail/u/0/?pli=1\%5Cnpapers3://publication/uuid/D84FC782E317-4880-B951-0697213436E1.

This article is protected by copyright. All rights reserved. 
4. Maslach C, Schaufeli WB, Leiter MP. Job Burnout. Annu Rev Psychol. 2001;52:397422.

5. Lee RT, Ashforth BE. A longitudinal study of burnout among supervisors and managers: comparisons between the Leiter and Maslach (1988) and Golembiewski et al. (1986) models. Organ Behav Hum Decis Process. 1993;54:369-398.

6. Taris TW, Le Blanc PM, Schaufeli WB, Schreurs PJG. Are there causal relationships between the dimensions of the Maslach Burnout Inventory? A review and two longitudinal tests. Work Stress. 2005;19(3):238-255.

doi:10.1080/02678370500270453.

7. Isoard-Gautheur S, Guillet-Descas E, Gaudreau P, Chanal J. Development of burnout perceptions during adolescence among high-level athletes: a developmental and gendered perspective. J Sport Exerc Psychol. 2015;37(4):436-448. doi:10.1123/jsep.2014-0251.

8. Martinent G, Louvet B, Decret J. Longitudinal trajectories of athlete burnout among young table tennis players: A 3-wave study. J Sport Heal Sci. 2016;(November). doi:10.1016/j.jshs.2016.09.003.

9. Leiter MP, Maslach C. The impact of interpersonal environment on burnout and organizational commitment. J Organ Behav. 1988;9(4):297-308. doi:10.1002/job.4030090402.

10. Schaufeli WB, Buunk BP. Burnout: An overview of 25 years of research and theorizing. In: Schabracq MJ, Winnubst JAM, Cooper CL, eds. Handbook of Work and Health Psychology. Chichester: Wiley; 2003:383-425.

11. Cordes CL, Dougherty TW, Blum M. Patterns of burnout among managers and 
professionals: A comparison of models. J Organ Behav. 1997;18(6):685-701. doi:10.1002/(SICI)1099-1379(199711)18:6<685::AID-JOB817>3.3.CO;2-L.

12. Hakanen JJ, Bakker AB, Schaufeli WB. Burnout and work engagement among teachers. J Sch Psychol. 2006;43(6):495-513. doi:10.1016/j.jsp.2005.11.001.

13. Maslach C, Jackson S. ., Leiter MP. Maslach Burnout Inventory: Manual. 3:rd ed. Paulo Alto: Consulting Psychologist Press; 1996.

14. Raedeke TD, Smith AL. Development and preliminary validation of an athlete burnout measure. J Sport Exerc Psychol. 2001;23(4):281-306. http://psycnet.apa.org/psycinfo/2001-09670-002. Accessed January 24, 2012.

15. Raedeke TD. Is Athlete Burnout More Than Just Stress? A Sport Commitment Perspective. J Sport Excercise Psychol. 1997;19(4):396-417.

16. Raedeke TD, Smith AL. Athlete Burnout Questionnaire Manual. 1st ed. Morganstown, VW; 2009.

17. Golembiewski RT, Munzenrider RF, Stevenson JG. Stress and Organizations: Toward a Phase Model of Burnout. New York: Praeger; 1986.

18. Grossi G, Perski A, Osika W, Savic I. Stress-related exhaustion disorder - clinical manifestation of burnout? A review of assessment methods, sleep impairments, cognitive disturbances, and neuro-biological and physiological changes in clinical burnout. Scand J Psychol. 2015;56(6):626-636. doi:10.1111/sjop.12251.

19. Gustafsson H, Hassmen P, Kenttä G, Johansson M. A qualitative analysis of burnout in elite Swedish athletes '. Psychol Sport Exerc. 2008;9(6):800-816.

20. Martinent G, Decret J-C, Guillet-Descas E, Isoard-Gautheur S. A reciprocal effects model of the temporal ordering of motivation and burnout among youth table tennis

This article is protected by copyright. All rights reserved. 
players in intensive training settings. J Sports Sci. 2014;32(17):1648-1658.

doi:10.1080/02640414.2014.912757.

21. Appleton PR, Hall HK, Hill AP. Relations between multidimensional perfectionism and burnout in junior-elite male athletes. Psychol Sport Exerc. 2009;10(4):457-465. doi:10.1016/j.psychsport.2008.12.006.

22. Maslach C, Jackson SE. The measurement of experienced burnout. J Organ Behav. 1981;2(2):99-113. doi:10.1002/job.4030020205.

23. Cresswell SL, Eklund RC. The convergent and discriminant validity of burnout measures in sport: a multi-trait/multi-method analysis. J Sports Sci. 2006;24(2):209220. doi:10.1080/02640410500131431.

24. Curran PJ, Howard AL, Bainter S a., Lane ST, McGinley JS. The separation of between-person and within-person components of individual change over time: A latent curve model with structured residuals. J Consult Clin Psychol. 2014;82(5):879894. doi:10.1037/a0035297.

25. Enders CK. Applied Missing Data Analysis. New York, NY: Guilford Press; 2010.

26. Marsh HW. Application of Confirmatory Factor Analysis and Structural Equation Modeling in Sport and Exercise Psychology. In: Tenenbaum G, Eklund RC, eds. Handbook of Sport Psychology. 3:rd ed. Hoboken, NJ: John Wiley \& Sons, Ltd; 2007:774-798.

27. Gustafsson H, Kenttä G, Hassmén P. Athlete burnout: an integrated model and future research directions. Int Rev Sport Exerc Psychol. 2011;4(1):3-24. doi:10.1080/1750984X.2010.541927.

28. Gustafsson H, Lundkvist E, Podlog L, Lundqvist C. Conceptual Confusion and 
Potential Advances in Athlete Burnout Research. Percept Mot Skills. 2016;(In press):1-11.

29. Bianchi R, Schonfeld IS, Laurent E. The Dead End of Current Research on Burnout Prevalence. J Am Coll Surg. 2016;223(2):424-425.

doi:10.1016/j.jamcollsurg.2016.05.012.

30. Binder M, Coad A. From Average Joe's happiness to Miserable Jane and Cheerful John: using quantile regressions to analyze the full subjective well-being distribution. $J$ Econ Behav Organ. 2011;79(3):275-290. doi:10.1016/j.jebo.2011.02.005.

31. Ivarsson A, Andersen MB. What counts as "evidence" in evidence-based practice? Searching for some fire behind all the smoke. J Sport Psychol Action. 2016;7(1):11-22. doi:10.1080/21520704.2015.1123206.

This article is protected by copyright. All rights reserved. 
Table 1. Within person relations between burnout subscales and between person slopes and intercepts for sample 1

\begin{tabular}{|c|c|c|c|c|c|c|c|c|c|c|c|c|c|c|}
\hline \multirow{2}{*}{$\begin{array}{l}\text { Exhasution } \rightarrow \text { Devaluation } \\
\text { Within person predictions }\end{array}$} & \multicolumn{2}{|l|}{$\mathrm{T} 1-2$} & \multicolumn{2}{|c|}{$\mathrm{T} 2-3$} & \multicolumn{2}{|l|}{ T3-4 } & \multicolumn{2}{|l|}{$\mathrm{T} 4-5$} & \multicolumn{2}{|l|}{ T5-6 } & \multicolumn{2}{|l|}{ T6-7 } & \multicolumn{2}{|l|}{$\mathrm{T} 7-8$} \\
\hline & $\beta$ & S.E & $\beta$ & S.E & $\beta$ & S.E & $\beta$ & S.E & $\beta$ & S.E & $\beta$ & S.E & $\beta$ & S.E \\
\hline Exhaustion $\rightarrow$ Exhaustion & $.27 * *$ & .09 & $.21 *$ & .09 & $.18 *$ & .07 & $.23 * *$ & .08 & $.24 * *$ & .10 & $.14 * *$ & .05 & $.26 * *$ & .10 \\
\hline Exhaustion $\rightarrow$ Devaluation & .04 & .12 & .04 & .07 & .05 & .05 & .08 & .07 & .09 & .08 & .10 & .09 & .09 & .09 \\
\hline Devaluation $\rightarrow$ Devaluation & .17 & .11 & .15 & .12 & .13 & .09 & .19 & .16 & .16 & .11 & .17 & .13 & .08 & .07 \\
\hline Devaluation $\rightarrow$ Exhaustion & .04 & .06 & .04 & .05 & .04 & .05 & .05 & .06 & .05 & .07 & .03 & .04 & .03 & .05 \\
\hline
\end{tabular}

Devaluation $\rightarrow$ Exhaustion

$.04 \quad .06$

Slope Exhaustion

Slope Devaluation

Intercept Exhaustion

Intercept Devaluation

$.01 \quad .01$

Model 2 Devaluation $\rightarrow$ RSA

Within person predictions

Devaluation $\rightarrow$ Devaluation

Devaluation $\rightarrow$ RSA

RSA $\rightarrow$ RSA

$2.02 \quad .05$

$1.79 \quad .05$

RSA $\rightarrow$ Devaluation

T1-2

T2-3

T3-4

$\begin{array}{ll}.17 & .13 \\ .06 & .07 \\ .11 & .07 \\ .07 & .06\end{array}$

Between person estimates

Slope Devaluation

Slope RSA

Intercept Devaluation

$.18 \quad .13$

$\begin{array}{ll}.18 & .13 \\ .09 & .10\end{array}$

$.15 \quad .09$

$.08 \quad .07$

.07

$\begin{array}{ll}.13 & .14 \\ .07 & .05 \\ .07 & .15 \\ .06 & .07\end{array}$

$\begin{array}{ll}.14 & .09 \\ .05 & .08 \\ .15 & .09 \\ .07 & .07\end{array}$

T4-5

$\mathrm{T} 7-8$

.09
.08
.09
.07

$\begin{array}{ll}.20 & .18 \\ .04 & .09 \\ .12 & .08 \\ .07 & .06\end{array}$

$\begin{array}{ll}.20 & .14 \\ .02 & .08 \\ .14 & .09 \\ .09 & .08\end{array}$

.15
.01
.13
.08

.11
.10
.08

10

.08

$.12 \quad .09$

$.02 \quad .12$

$\begin{array}{ll}.02 & .06\end{array}$

Model 3 Exhaustion --> RSA $\quad$ T1-2

$2.42 \quad .06$

Within person prediction

Exhaution $\rightarrow$ Exhaustion

Exhaution $\rightarrow$ RSA

RSA $\rightarrow$ RSA

$28 * *$

$\mathrm{T} 2-3$

$\mathrm{T} 2-3$

T5-6

T6-7

T7-8

$\begin{array}{llll}.22 * & .10 & .20 * \quad .08\end{array}$

RSA $\rightarrow$ Exhaustion

$\begin{array}{ll}.28 * * & .10 \\ .09 & .11\end{array}$

.10
.11

.07

.06

.10

.08
.06

$.27 * *$

$.27 * *$
.14

.14
.11

.10

$27 *$

$\begin{array}{ll}.27 * & .11 \\ .13 & .08\end{array}$

.01

.05

.01

.05

.01

Slope Exhaustion

Slope RSA

$.00 \quad .01$

$.02 * * \quad 01$

$2.02 \quad .05$

tercept Exhaustion

2.41

Intercept RSA

.06

$* P<0.05 ; * * P<0.01 ; * * * P<0.001$.

This article is protected by copyright. All rights reserved. 
Table 2. Within person relations between burnout subscales and between person slopes and intercepts for sample 2

\begin{tabular}{|c|c|c|c|c|c|c|}
\hline \multirow{2}{*}{$\begin{array}{l}\text { Model } 1 \text { Exhaustion } \rightarrow \text { Devaluation } \\
\text { Within person predictions }\end{array}$} & \multicolumn{2}{|l|}{$\mathrm{T} 1-2$} & \multicolumn{2}{|l|}{$\mathrm{T} 2-3$} & \multicolumn{2}{|l|}{ T3-4 } \\
\hline & $\beta$ & S.E & $\beta$ & S.E & $\beta$ & S.E \\
\hline Exhaustion $\rightarrow$ Exhaustion & -.07 & .13 & -.05 & .11 & -.06 & .12 \\
\hline Exhaustion $\rightarrow$ Devaluation & $-.30 *$ & .14 & -.04 & .11 & .17 & .21 \\
\hline Devaluation $\rightarrow$ Devaluation & .06 & .14 & .08 & .17 & .09 & .20 \\
\hline Devaluation $\rightarrow$ Exhaustion & .03 & .09 & .04 & .12 & .05 & .14 \\
\hline \multicolumn{7}{|l|}{ Between person estimates } \\
\hline Slope Exhaustion & $.08 * *$ & .03 & & & & \\
\hline Slope Devaluation & $.07 * *$ & .03 & & & & \\
\hline Intercept Exhaustion & 2.25 & .08 & & & & \\
\hline Intercept Devaluation & 1.80 & .08 & & & & \\
\hline Model 2 Devaluation $\rightarrow$ Reduced Sport Accomplishment & T1-2 & & $\mathrm{T} 2-3$ & & T3-4 & \\
\hline \multicolumn{7}{|l|}{ Within person predictions } \\
\hline Devaluation $\rightarrow$ Devaluation & -.01 & .14 & -.01 & .22 & -.02 & .28 \\
\hline Devaluation $\rightarrow$ RSA & .04 & .14 & .06 & .14 & .05 & .19 \\
\hline $\mathrm{RSA} \rightarrow \mathrm{RSA}$ & -.02 & .12 & -.02 & .14 & -.02 & .14 \\
\hline RSA $\rightarrow$ Devaluation & .20 & .13 & .02 & .11 & .22 & .13 \\
\hline \multicolumn{7}{|l|}{ Between person estimates } \\
\hline Slope Devaluation & $.08 * *$ & .02 & & & & \\
\hline Slope Reduced Sense of Accomplishment & .00 & .02 & & & & \\
\hline Intercept Devaluation & 1.79 & .08 & & & & \\
\hline Intercept Reduced Sense of Accomplishment & 2.47 & .07 & & & & \\
\hline Model 3 Exhaustion $\rightarrow$ Reduced Sport Accomplishment & T1-2 & & T2-3 & & T3-4 & \\
\hline \multicolumn{7}{|l|}{ Within person predictions } \\
\hline Exhaustion $\rightarrow$ Exhaustion & -.06 & .11 & -.06 & .10 & -.06 & .12 \\
\hline Exhaustion $\rightarrow$ RSA & -.11 & 14 & -.03 & .10 & .07 & .18 \\
\hline $\mathrm{RSA} \rightarrow \mathrm{RSA}$ & -.10 & .10 & -.14 & .17 & -.13 & .14 \\
\hline RSA $\rightarrow$ Exhaustion & .05 & .07 & .06 & .08 & .06 & .09 \\
\hline \multicolumn{7}{|l|}{ Between person estimates } \\
\hline Slope Exhaustion & $.07 *$ & .03 & & & & \\
\hline Slope Reduced Sense of Accomplishment & -.07 & .02 & & & & \\
\hline Intercept Exhaustion & 2.26 & .09 & & & & \\
\hline Intercept Reduced Sense of Accomplishment & 2.48 & .08 & & & & \\
\hline
\end{tabular}

$* P<0.05 ; * * P<0.01 ; * * * P<0.001$.

This article is protected by copyright. All rights reserved. 
Figure 1. Theoretical LCM-USR model with cross lagged residuals

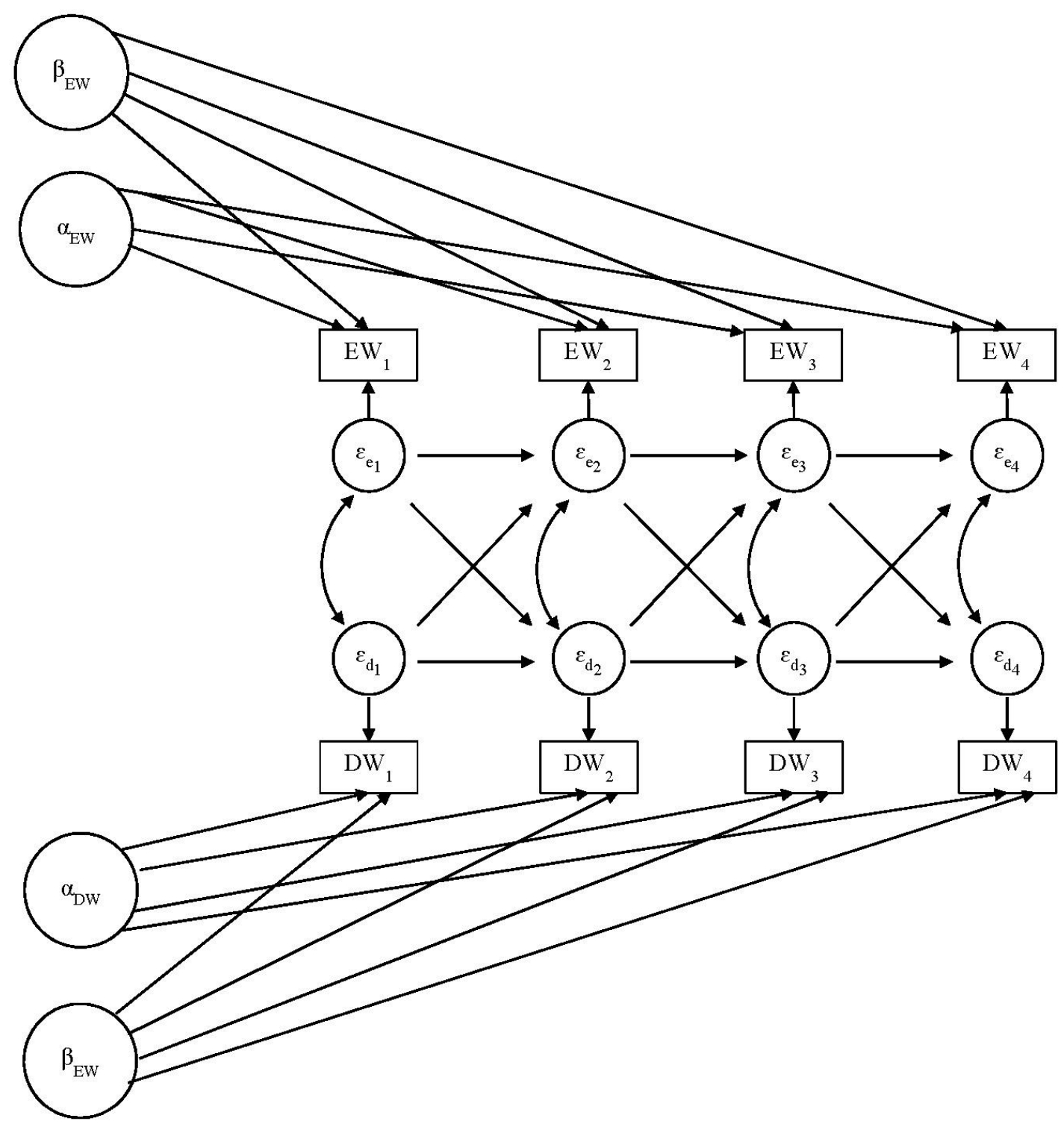

This article is protected by copyright. All rights reserved. 